\title{
TEMPERATURE DEPENDENCE OF MAGNETIC INELASTIC NEUTRON SCATTERING IN $\mathrm{Mn}(12 \% \mathrm{Ge})$ ALLOY
}

\author{
J. JaNKowSKa-Kisielińska, K. MikKe, J.J. MiLCZAREK \\ Institute of Atomic Energy, Świerk, 05-400 Otwock, Poland
}

\section{B. HENNION}

Laboratoire Léon Brillouin, CEN Saclay, France

\section{AND V.A. UDOVENKO}

Institute of Physical Metallurgy, Moscow, Russia

\begin{abstract}
A systematic study of temperature dependence of magnetic inelastic neutron scattering for itinerant antiferromagnet $\mathrm{Mn}(12 \% \mathrm{Ge})$ is presented. At low temperatures the experimental data are well described in terms of damped spin waves. This description becomes insufficient at least $150 \mathrm{~K}$ below $T_{\mathrm{N}}$. In the temperature range $200-400 \mathrm{~K}$ the integrated intensity of distribution for energy transfer $13 \mathrm{THz}$ increases by one third and the shape of the deconvoluted neutron scattering cross-section changes. These changes suggest that $c a .200 \mathrm{~K}$ below $T_{\mathrm{N}}$ an additional contribution to the inelastic neutron scattering starts to appear and that its origin is different from spin waves.
\end{abstract}

PACS numbers: 75.30.Ds, 75.50.Ee

The magnetic excitations in itinerant antiferromagnets are not yet satisfactorily understood. In particular more detailed studies of their temperature dependence are needed. The aim of our experiment was to study the evolution of the neutron scattering by spin waves at low temperature into the paramagnetic scattering above $T_{\mathrm{N}}$.

The crystal structure of the $\gamma$-Mn alloys with high manganese concentration (fcc in the paramagnetic phase) becomes tetragonal with $c<a$ in the antiferromagnetic (AF) phase. Many alloys with lower manganese concentration remain fcc in the AF phase. In some narrow concentration region (different for different diluents) two other crystal structures: orthorhombic and tetragonal with $c>a$ appear in the AF phase. The composition of the investigated alloy is close to the composition for the tricritical point for paramagnetic fcc, AF tetragonal and AF 
orthorhombic phases. At room temperature it is antiferromagnet with tetragonal structure. Tetragonal deformation is $1-c / a=0.013$. The estimated Néel temperature is $470-490 \mathrm{~K}$. The investigated sample is a polydomain single-crystal with the mosaic spread $c a .2^{\circ}$.

The measurements were performed using a 3-axis neutron spectrometer at the Orphèe reactor in LLB CEN Saclay. The scattered neutron energy was constant $E_{f}=3.55 \mathrm{THz}$. The horizontal collimations starting from the reactor core were $26^{\prime}-30^{\prime}-60^{\prime}-60^{\prime}$. The intensity distributions versus momentum transfer for energy transfers 5,8 and $13 \mathrm{THz}$ were measured in the temperature range 19-600 K. At temperatures 19 and $295 \mathrm{~K}$ additional distributions were measured to obtain more complete data on the dispersion relation. The energy distribution of the incoherent scattering was measured far from the Brillouin zone (BZ) center at $19 \mathrm{~K}$. It was used to estimate the incoherent scattering at higher temperatures. Examples of the intensity distributions measured at $19 \mathrm{~K}$ are presented in Fig. 1. The solid lines in the figure show the results of the computed convolution of the spectrometer resolution function with the neutron scattering cross-section for the damped oscillator model. The dashed lines indicate the background plus incoherent scattering level.

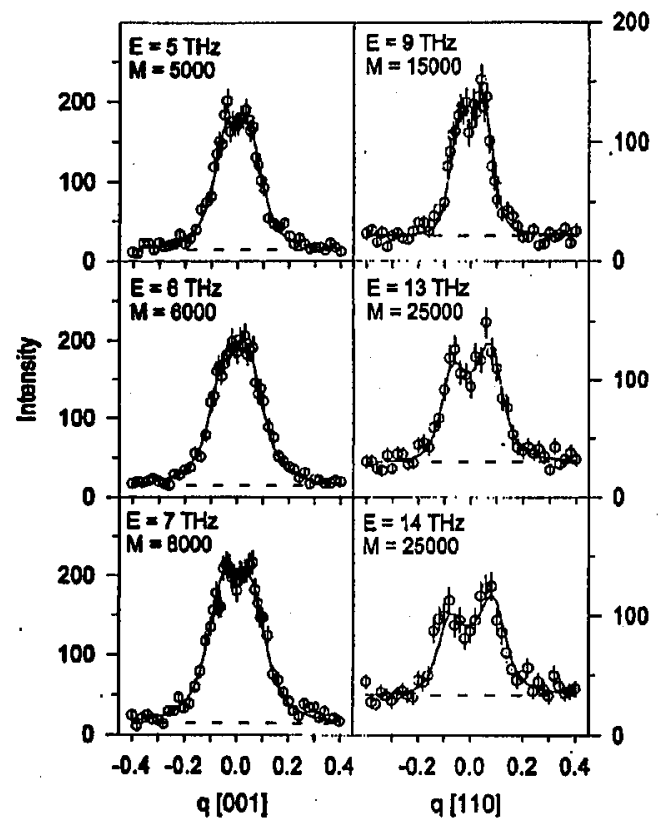

Fig. 1. The intensity distributions versus momentum transfer for energy transfers 5,6 and $7 \mathrm{THz}$ measured in [001] direction and for 9, 13 and $14 \mathrm{THz}-$ in [110] direction at $19 \mathrm{~K}$. 
For data analysis below $T_{N}$ we used the neutron scattering cross-section for damped spin waves. Two formulae were used: the one for itinerant antiferromagnet (Liu [1], Sato and Maki [2]), which reads as follows:

$$
\frac{\mathrm{d}^{2} \sigma}{\mathrm{d} \Omega \mathrm{d} E} \simeq F^{2}(Q)[n(E)+1] \frac{\Gamma E}{\left(E^{2}-E_{q}^{2}\right)^{2}+\Gamma^{2} E^{2}}
$$

and another one, for a damped harmonic oscillator (used for $\mathrm{Mn}(10 \% \mathrm{Cu})$ [3]):

$$
\frac{\mathrm{d}^{2}}{\mathrm{~d} \Omega \mathrm{d} E} \simeq F^{2}(Q)[n(E)+1] \frac{\Gamma E}{\left(E^{2}-E_{q}^{2}\right)^{2}+\Gamma^{2} E^{2}} \frac{\kappa^{2}}{\kappa^{2}+q^{2}},
$$

where $n(E)$ is the population factor, $F(Q)$ - magnetic form factor, $\kappa$ - inverse correlation range, $q$ - momentum reduced to the magnetic BZ. The spin wave energy follows the formula

$$
E_{q}^{2}=v^{2} q^{2}+E_{\mathrm{g}}^{2}
$$

where $v$ is the spin wave velocity and $E_{\mathrm{g}}$ - the energy gap in the spin wave spectrum. The spin wave damping parameter $\Gamma$ in these alloys has a linear dependence on the momentum $q$

$$
\Gamma=\Gamma_{0}+\Gamma_{1} q .
$$

The parameters of the dispersion relation $v$ and $E_{\mathrm{g}}$ and of the damping $\Gamma_{\mathrm{1}}$ were obtained by computer fitting of the intensity distributions. Goodness of fits for $19 \mathrm{~K}$ is slightly better for the second formula. The obtained shape of the cross-section is nearly independent on the used formula. The mean values of parameters for the first formula are: $v=48 \pm 6 \mathrm{THz} \AA, E_{\mathrm{g}}=1.96 \pm 0.04 \mathrm{THz}$ and $\Gamma_{1}=38 \pm$ $13 \mathrm{THz} \AA$. For the second formula the mean values are $v=54 \pm 4 \mathrm{THz} \AA$ and $\Gamma_{1}=39 \pm 20 \mathrm{THz} \AA$ for $\kappa=0.1 \AA^{-1}$. At temperatures 100 and $200 \mathrm{~K}$ only 3 scans were performed for energy transfers 5,8 and $13 \mathrm{THz}$. The obtained values of the parameters differ slightly, the velocity decreases and the damping increases. At $300-350 \mathrm{~K}$ a sudden increase in damping parameter is observed. The increase of damping is, however, not sufficient to describe the change of the shape of the measured distributions in terms of any of the above formulae. The quality of fits decreases drastically; the parameter $\chi^{2}$ is smaller than 2 only for 13 and $14 \mathrm{THz}$. Above $300 \mathrm{~K}$ fits for all measured distributions are rather poor $\left(\chi^{2}\right.$ usually bigger than 1.5). Above $T_{\mathrm{N}}$ the analysis in terms of paramagnetic fluctuations [4] is not yet completed.

The integrated intensity of the distribution versus momentum for energy $13 \mathrm{THz}$ divided by the population factor is shown in Fig. 2. One observes the increase in the integrated intensity by about one third in the temperature range $200-375 \mathrm{~K}$. Above $400 \mathrm{~K}$ there is a slow decrease. The main change takes place in a rather narrow temperature range and, surprisingly, much below $T_{\mathrm{N}}$. The deconvoluted neutron scattering cross-section derived using the first formula from distributions for $13 \mathrm{THz}$ for different temperatures is shown in Fig. 3. The shapes for 19 and $200 \mathrm{~K}$ are very similar, for 350 and $400 \mathrm{~K}$ they are also similar to each other but quite different from the first two. The maxima are shifted towards the center, the distributions are broader and they go very slowly to 0 for a larger $q$. The shape for $300 \mathrm{~K}$ is intermediate. The changes in the intensity and shape of the 

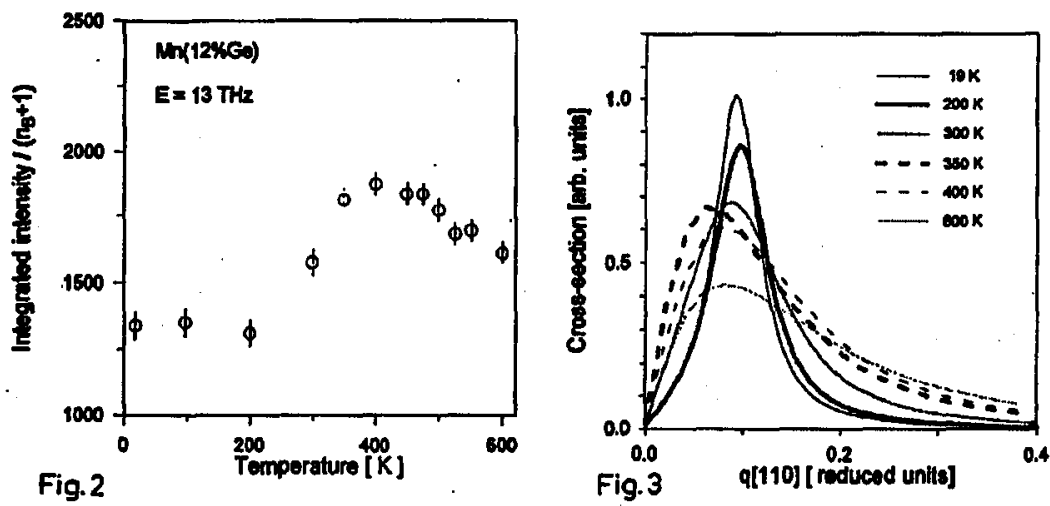

Fig. 2. The temperature dependence of the integrated intensity of the distribution for energy transfer $13 \mathrm{THz}$ corrected for the background, the incoherent scattering and the population factor.

Fig. 3 The neutron scattering cross-section divided by the population factor, derived using formula (1) from distributions for $13 \mathrm{THz}$ measured for different temperatures.

measured distributions suggest that $c a .200 \mathrm{~K}$ below $T_{\mathrm{N}}$ an additional contribution to the inelastic neutron scattering starts to appear and that its origin is different from spin waves.

This work was partly supported by the "Human Capital and Mobility Access to Large Scale Facilities PECO Extention" Program (contract No. ERB CIPD CT 940080).

\section{References}

[1] S.H. Liu, Phys. Rev. B 2, 2664 (1970).

[2] H. Sato, K. Maki, Int. J. Magnetism 6, 183 (1974).

[3] J.A. Fernandez-Baca, M.E. Hagen, R.M. Nicklow, T.G. Perring, Y. Tsunoda, J. Appl. Phys. 73, 6548 (1993).

[4] A. Yazaki, K. Tajima, Y. Todate, S. Tomiyoshi, H. Ikeda, J. Phys. Soc. Jpn. 63, 748 (1994). 